\title{
Association between Faecalibacterium prausnitzii Reduction and Inflammatory Bowel Disease: A Meta-Analysis and Systematic Review of the Literature
}

\author{
Yuan Cao, Jun Shen, and Zhi Hua Ran \\ Renji Hospital, School of Medicine, Shanghai Jiao Tong University, Shanghai Institution of Digestive Disease, \\ Shanghai Inflammatory Bowel Disease Research Center, Shanghai 200127, China
}

Correspondence should be addressed to Zhi Hua Ran; zhihuaran@vip.163.com

Received 31 December 2013; Accepted 17 February 2014; Published 27 March 2014

Academic Editor: Paolo Gionchetti

Copyright (C) 2014 Yuan Cao et al. This is an open access article distributed under the Creative Commons Attribution License, which permits unrestricted use, distribution, and reproduction in any medium, provided the original work is properly cited.

Background. Laboratory data suggests a reduction of Faecalibacterium prausnitzii (F. prausnitzii) is confirmed both in fecal samples in inflammatory bowel disease (IBD) patients. Numerous observational studies have suspected dysbiosis, an imbalance between protective and harmful bacteria to be relevant to the etiology and pathogenesis of IBD. Methods. Medline, EMBASE, Pubmed, and others. were searched by 2 independent reviewers. Of 48 abstracts reviewed, 11 studies met our inclusion criteria (subject $N=1180$ ). Meta-analysis was performed with Review Manager 5.2. Results. The bacterial count of $F$. prausnitzii in IBD patients was significantly lower $(6.7888 \pm 1.8875) \log 10 \mathrm{CFU} / \mathrm{g}$ feces than healthy controls $(7.5791 \pm 1.5812) \log 10 \mathrm{CFU} / \mathrm{g}$ feces; $P<0.0001$. The Standardization Mean Difference of $F$. prausnitzii in IBD patients was -0.94 (95\% confidence interval [CI]: $-1.07--0.80)$. Subgroup analyses revealed a trend toward a greater effect for CD (SMD: -1.13 , 95\% CI: $-1.32--0.94)$ when compared to UC (SMD: -0.78 , 95\% CI: -0.97--0.60). Conclusions. The abundance of $F$. prausnitzii was decreased in IBD patients compared with healthy controls. Furthermore, the reduction of F. prausnitzii and misbalance of the intestinal microbiota are particularly higher in CD patients with ileal involvement.

\section{Introduction}

IBD is suspected to arise from the interaction between the host's genetic background, mucosal immunity, and the resident bacterial flora [1]. Genome-wide association studies (GWAS) have identified more than 160 host genetic variants. Many are related to human gut microbiota [2]. In patients with inflammatory bowel diseases (IBD), the composition and diversity of the microbiota are always altered [3]. The imbalance between potentially "beneficial" and potentially "harmful" bacteria, also called dysbiosis, plays a role in the pathogenesis of chronic mucosal inflammatory lesions of IBD [4].

F. prausnitzii belongs to the phylum of Firmicutes and is the major bacterium of the Clostridium leptum group. The Meta-analysis of the Human Intestinal Tract project have shown that F. prausnitzii is one of the most abundant anaerobic bacteria in the human gut microbiota, with a proportion of around 5\% of total bacteria in faeces [5]. F. prausnitzii plays an important role in providing energy to the colonocytes and maintaining the intestinal health [6]. Furthermore, there is emerging laboratory evidence illustrating a strong antiinflammatory effect of $F$. prausnitzii both in vitro and in vivo. And deficiency of $F$. prausnitzii might provoke and enhance inflammation [7]. Specially, a significant inverse correlation between disease activity and the count of $F$. prausnitzii was found in UC patients, even with quiescent disease [8]. The depletion of $F$. prausnitzii was observed in patients with untreated $\mathrm{CD}$ but not in the patients with chronic diarrhea, suggesting a relationship in the pathomechanisms of CD [9]. Finally, a diminished prevalence and abundance of $F$. prausnitzii are revealed in the fecal samples of patients with 
IBD. The F. prausnitzii level was much lower when the disease activity increased [10].

To further investigate the possible association between F. prausnitzii reduction and IBD, we conducted a metaanalysis and systematic review to estimate the relative risk of $F$. prausnitzii reduction in patients with and without IBD. Given the laboratory data previously cited, we hypothesized a relationship between $F$. prausnitzii reduction and IBD.

\section{Materials and Methods}

2.1. Search Strategy. This review was performed according to the standard guidelines for meta-analyses and systematic reviews of observational studies [11]. To find relevant articles for this review, we searched the following databases (from inception to November 2013): EMBASE, MEDLINE, Google Scholar, Pubmed, ACP Journal Club, the Cochrane Central Register of Controlled Trials, CMR, DARE, and HTA. The search strategy used free-text words to increase the sensitivity of the search. The following search terms were used: "inflammatory bowel disease," "Crohn's disease," "ulcerative colitis," "IBD," "UC," "CD," "Faecalibacterium prausnitzii," "F. prausnitzii," and "FP." Boolean operators (AND, OR, NOT) were used to widen and narrow the search results. The titles and abstracts from the search results were examined for potential inclusion. Also, the references from selected articles were examined as further search tools.

2.2. Study Selection. For inclusion in the systematic review, a study had to meet the following criteria established by the study team: (1) F. prausnitzii counts measured by polymerase chain reaction-denaturing gradient gel electrophoresis (PCRDGGE), terminal restriction fragment length polymorphism (T-RFLP), or fluorescence in situ hybridization (FISH), (2) studies of human, (3) inclusion of a control group, (4) IBD and control groups were similar in age and sex and from the same catchment area, and (5) data were reported that were sufficient to calculate $F$. prausnitzii reduction in both the IBD and control groups. Studies were excluded if they used data from a previously published study.

2.3. Data Extraction. To reduce reporting error and bias in data collection, 2 independent reviewers extracted data from selected studies using standardized data extraction forms. These forms, created by the study team, included the (a) title, (b) authors, (c) journal, (d) year of publication, (e) study design, (f) inclusion and exclusion criteria, (g) methods by which IBD was diagnosed, (h) methods by which $F$. prausnitzii reduction was diagnosed, (i) number of patients with ulcerative colitis (UC), (j) number of patients with Crohn's disease (CD), (k) number of patients in the control group, (l) reported previous use of antibiotics, probiotics, or prebiotics in the IBD and control groups, and $(\mathrm{m})$ reported previous use of steroids, 5-aminosalicylates (5-ASAs), and tumor necrosis factor-alpha (TNF- $\alpha$ ) antibody medications in the IBD group. Studies were excluded if participants had used steroids, 5-ASAs, TNF- $\alpha$ antibody antibiotics, probiotics, or prebiotics in the last month preceding fecal

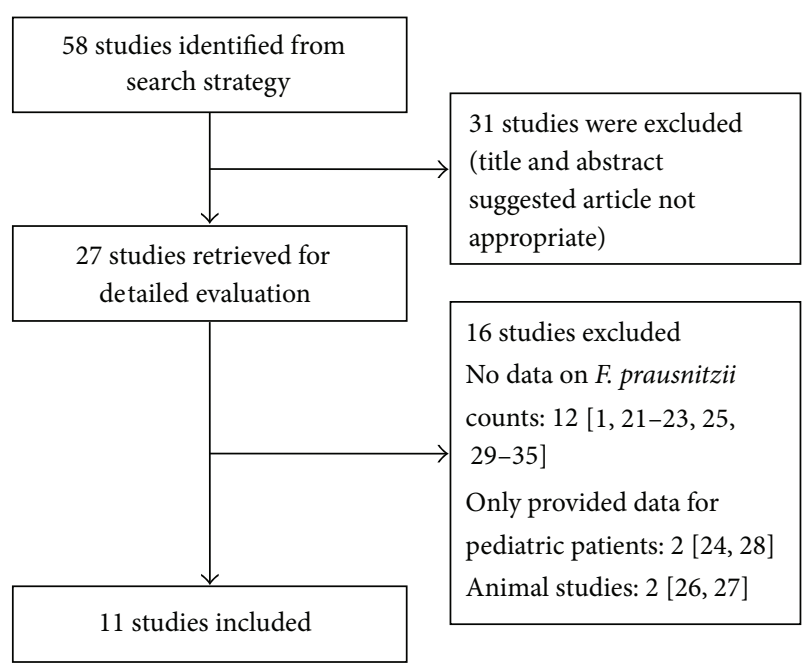

FIGURE 1: Flow diagram of studies identified in the systematic review.

sampling as this could influence the intestinal microbiota. If needed, authors were contacted regarding specific questions relating to their study. The independent reviewers conferred after data extraction was complete, discrepancies were identified, and review of the relevant article led to consensus.

2.4. Statistical Analysis. The primary outcome of this analysis was the Standardization Mean Difference (SMD) of F. prausnitzii counts in IBD versus controls. Std. mean difference was used to describe the counts of the F prausnitzii in IBD patients versus the controls. We calculated the SMD with a 95\% confidence interval (CI) based on a random-effects model as described by Mantel-Haenszel. Meta-analysis was performed with the Review Manager 5.2. Analysis with a funnel plot used to assess publication bias. An $I^{2}$ statistic was used to measure the proportion of inconsistency in individual studies that could not be explained by chance. Any heterogeneity identified would prompt subgroup analysis in an attempt to explain these findings.

2.5. Assessment of Study Quality. Each study chosen for review was carefully assessed for study quality by the study team. Study quality was assessed using the following criteria: (1) study design, (2) method of IBD diagnosis, (3) method of patient enrollment (consecutive versus selected), (4) method of F. prausnitzii counts measurement, and (5) whether $F$. prausnitzii reduction was the primary or secondary outcome of the study.

\section{Results}

3.1. Search Results. Our initial search strategy yielded 58 potential articles for inclusion. After detailed analysis of selected articles, 27 articles were reviewed in detail. Subsequently, 16 articles did not meet inclusion criteria [1, 21-35]. The reasons for exclusion included: 12 studies did not provide data on $F$. prausnitzii counts [1, 21-23, 25, 29-35]. 2 studies 
TABLE 1: Characteristics of the included studies.

\begin{tabular}{|c|c|c|c|c|c|c|c|c|c|}
\hline Author & Year & Location & $\begin{array}{c}\text { Single versus } \\
\text { multicenter }\end{array}$ & $n$, total & $\begin{array}{c}n, \mathrm{IBD} \\
(\mathrm{CD} / \mathrm{UC})\end{array}$ & $n$, control & $\begin{array}{c}\text { Control } \\
\text { composition }\end{array}$ & $\begin{array}{c}\text { Mean age, IBD } \\
(\mathrm{CD} / \mathrm{UC})\end{array}$ & $\begin{array}{c}\text { Mean age, } \\
\text { control }\end{array}$ \\
\hline Machiels et al. [8] & 2013 & Belgium & Single & 214 & $0 / 127$ & 87 & Healthy controls & 43 & 41.5 \\
\hline Varela et al. [12] & 2013 & Spain & Single & 176 & $0 / 116$ & 31 & Healthy controls & 40 & 32 \\
\hline Swidsinski et al. [13] & 2008 & Germany & Single & 422 & $82 / 105$ & 32 & Healthy controls & $35 / 41$ & 40 \\
\hline Dörffel et al. [9] & 2012 & Germany & Single & 171 & $50 / 0$ & 25 & Healthy controls & 39 & 48 \\
\hline $\begin{array}{l}\text { Vermeiren et al. } \\
\text { [14] }\end{array}$ & 2012 & Belgium & Single & 12 & $0 / 6$ & 6 & Healthy controls & Not reported & Not reported \\
\hline Joossens et al. [15] & 2011 & Belgium & Single & 207 & $68 / 0$ & 55 & Healthy controls & Not reported & Not reported \\
\hline Wang et al. [16] & 2013 & China & Single & 76 & $21 / 34$ & 21 & Healthy controls & Not reported & Not reported \\
\hline Jia et al. [17] & 2010 & UK & Single & 73 & $20 / 14$ & 18 & Healthy controls & Not reported & Not reported \\
\hline Sokol et al. [18] & 2009 & France & Single & 133 & $22 / 13$ & 27 & Healthy controls & $37 / 40$ & 36 \\
\hline Andoh et al. [19] & 2012 & Japan & Multicenter & 188 & $67 / 0$ & 121 & Healthy controls & 30 & 32 \\
\hline Willing et al. [20] & 2009 & Sweden & Single & 20 & $6 / 0$ & 6 & Healthy controls & Not reported & Not reported \\
\hline
\end{tabular}

IBD: inflammatory bowel disease; CD: Crohn's disease; UC: ulcerative colitis.

TABLE 2: Quality assessment of the included studies.

\begin{tabular}{|c|c|c|c|c|c|c|}
\hline Author & $\begin{array}{l}\text { F. prausnitzii } \\
\text { counts }\end{array}$ & IBD diagnosis & Study type & $\begin{array}{c}\text { Patient } \\
\text { enrollment }\end{array}$ & Outcome & Samples \\
\hline Machiels et al. [8] & RT-PCR & Not reported & Retrospective & Not reported & Primary & Stools \\
\hline Varela et al. [12] & RT-PCR & $\begin{array}{c}\text { Colonoscopy and } \\
\text { histology }\end{array}$ & Retrospective & Not reported & Primary & Stools \\
\hline Swidsinski et al. [13] & FISH & Colonoscopy & Retrospective & Not reported & Primary & Stools \\
\hline Dörffel et al. [9] & FISH & Colonoscopy & Retrospective & Not reported & Primary & Stools \\
\hline Vermeiren et al. [14] & RT-PCR & Not reported & Retrospective & Not reported & Primary & Stools \\
\hline Joossens et al. [15] & RT-PCR & Not reported & Retrospective & Not reported & Primary & Stools \\
\hline Wang et al. [16] & RT-PCR & Not reported & Retrospective & Not reported & Primary & Biopsies \\
\hline Jia et al. [17] & RT-PCR & Not reported & Retrospective & Not reported & Primary & Stools \\
\hline Sokol et al. [18] & RT-PCR & Not reported & Retrospective & Not reported & Primary & Stools \\
\hline Andoh et al. [19] & T-RFLP & Not reported & Retrospective & Not reported & Primary & Stools \\
\hline Willing et al. [20] & RT-PCR & Colonoscopy & Retrospective & Not reported & Primary & Biopsies \\
\hline
\end{tabular}

only provided data for pediatric patients [24, 28]. 2 studies were animal studies $[26,27]$. Therefore, 11 studies $[8,9,12-$ 20] with 1180 patients fulfilled the inclusion criteria for the review (Figure 1).

3.2. Study Characteristics. The characteristics of the included studies are summarized in Tables 1 and 2 . The results of each study are in Table 3. F. prausnitzii counts were expressed as $\log 10$ values per gram feces. The largest and earliest study examining the relationship between $F$. prausnitzii reduction and IBD was conducted in Germany by Swidsinski et al. [13]. The authors investigated sections of paraffinembedded punched fecal cylinders using fluorescence in situ hybridization (FISH). F. prausnitzii with high concentration was counted within a $10 * 10 \mu \mathrm{m}$ area of the microscopic field representative of the region of interest. F. prausnitzii with uneven distribution or overall low concentrations was enumerated within larger areas of $100 * 100 \mu \mathrm{m}$.

8 of the included studies confirmed the differences in the presence or intensity of $F$. prausnitzii counts after denaturing gradient gel electrophoresis (DGGE) by real-time PCR (RTPCR) $[8,12,14-18,20]$. F prausnitzii cannot be cultured owing to its requirement for a complex anaerobic environment [19]. By RT-PCR, they were able to amplify, clone, and sequence the bacterial $16 \mathrm{~S}$ ribosomal RNA genes and analyze the fecal samples individually to avoid the possible error [36].

Three of the included studies commented on the activity of IBD and F. prausnitzii counts. Wang et al. found sharply decreased $F$. prausnitzii in the feces of active CD and UC patients [16]. Sokol et al. and Andoh et al. reported lower counts of $F$. prausnitzii in active CD patients compared to CD patients in remission $[18,19]$. 
TABLE 3: Study results.

\begin{tabular}{|c|c|c|c|}
\hline Author & $\begin{array}{c}\log 10 \text { copies/g } \\
\text { IBD patients } \\
(\mathrm{CD} / \mathrm{UC})\end{array}$ & $\begin{array}{l}\log 10 \text { copies/g } \\
\text { healthy controls }\end{array}$ & $P$ \\
\hline Machiels et al. [8] & $0 /(10.95 \pm 1.41)$ & $11.72 \pm 1.08$ & $<0.0001$ \\
\hline Varela et al. [12] & $0 /(8.02 \pm 0.57)$ & $8.90 \pm 0.37$ & $<0.0001$ \\
\hline Swidsinski et al. [13] & $(9.75 \pm 9.77) /(10.14 \pm 10.02)$ & $10.17 \pm 9.65$ & $<0.0001$ \\
\hline Dörffel et al. [9] & $(9.06 \pm 9.33) / 0$ & $10.21 \pm 9.94$ & $<0.001$ \\
\hline Vermeiren et al. [14] & $0 /(5.56 \pm 0.83)$ & $6.63 \pm 0.95$ & 0.07 \\
\hline Joossens et al. [15] & $(9.44 \pm 1.85) / 0$ & $10.97 \pm 1.25$ & $<0.0001$ \\
\hline Wang et al. [16] & $(0.03 \pm 0.06) /(0.23 \pm 0.51)$ & $1.40 \pm 1.06$ & $<0.0001$ \\
\hline Jia et al. [17] & $(5.71 \pm 5.34) /(5.93 \pm 5.87)$ & $5.93 \pm 5.83$ & $<0.05$ \\
\hline Sokol et al. [18] & $(8.81 \pm 0.52) /(8.70 \pm 0.63)$ & $10.4 \pm 0.2$ & 0.0004 \\
\hline Andoh et al [19] & $(0.40 \pm 0.09) / 0$ & $0.81 \pm 0.04$ & $<0.0001$ \\
\hline Willing et al. [20] & $(0.40 \pm 0.89) / 0$ & $8.72 \pm 2.49$ & $<0.001$ \\
\hline
\end{tabular}

1 of the included studies examined the F. prausnitzii counts before and after treatment by an element diet [17]. It suggests recovery following elemental diet is attributed to lower levels of gut flora.

1 of the included studies reported the relationship between the maintenance of clinical remission and the recovery of the $F$. prausnitzii population after relapse Varela et al. found low counts of $F$. prausnitzii were associated with less than 12 months of remission and more than 1 relapse/year [12].

3.3. Meta-Analysis of SMD. Overall, the bacterial count of $F$. prausnitzii in IBD patients was significantly lower $(6.7888 \pm$ 1.8875) $\log 10 \mathrm{CFU} / \mathrm{g}$ feces than healthy controls $(7.5791 \pm$ 1.5812) $\log 10 \mathrm{CFU} / \mathrm{g}$ feces; $P<0.0001$. The SMD of F. prausnitzii in IBD patients was -0.94 (95\% confidence interval [CI]: $-1.07--0.80$ ) (Figure 2). Subgroup analyses revealed a trend toward a greater effect for CD (SMD: -1.13 , 95\% CI: $-1.32--0.94$ ) when compared to UC (SMD: -0.78 , 95\% CI: -0.97--0.60). There was significant heterogeneity in the included studies $\left(I^{2}=96 \%\right)$. Furthermore, analysis of the funnel plots for publication bias suggested a possible bias against small studies demonstrating high SMD (Figure 3).

\section{Discussion}

Our systematic review and meta-analysis of the literature has identified recent studies examining the relationship between F. prausnitzii reduction and IBD. The majority of recent studies find a higher rate of $F$. prausnitzii reduction in IBD patients as compared to controls. All of the 11 included studies found significantly lower F. prausnitzii counts in IBD patients versus controls. Our meta-analysis suggests a possible link with the reduction of $F$. prausnitzii andmisbalance of the intestinal microbiota and IBD patients, especially CD patients with ileal involvement. The levels of F. prausnitzii were extremely low in two studies [19, 28]. Wang et al. took biopsies samples from active CD patients and found extremely lower F. prausnitzii counts compared to stools [28]. Andoh et al. demonstrated a difference in gut microbiota of the Japanese population, suggesting that environmental factors such as sanitation, diet, hygiene, and ethnicity were important for shaping the gut microbiota [19]. However, significant heterogeneity and the possibility of publication bias limit our certainty in this association. Furthermore, Hansen et al. challenged the current model of a protective role for F. prausnitzii in CD [24]. They reported an increasein mucosal Faecalibacterium in pediatric CD patients compared with controls, which suggested a more dynamic role for this organism than previously described in adult IBD. It is possible that the microbial signature of pediatric IBD is distinct from adult disease. Furthermore, the early host and microbiota response to IBD may induce proliferation of F. prausnitzii to reverse the inflammatory change, which still remains to be explained.

Mechanistic theories of microbial etiopathogenesis between the possible protective benefit of $F$. prausnitzii against IBD have been proposed. Duncan et al. demonstrated that the major end products of glucose fermentation by $F$. prausnitzii strains are substantial quantities of butyrate [37]. Butyrate plays a major role in gut physiology, protection against pathogen invasion, and modulation of immune system [38]. Butyrate is the primary energy source for intestinal epithelial cells, which are fundamental elements for the maintenance of barrier integrity [26]. Therefore, butyrate may contribute to the anti-inflammatory effect. Additionally, butyrate may inhibit inflammatory response through inhibition of histone deacetylase activity, resulting in suppression of NF- $\kappa \mathrm{B}$ activity and hyperacetylation of histones [12]. Furthermore, Himmel et al. found that $F$. prausnitzii could induce relatively low amounts of IL-12 and large amounts of IL-10 and Tregs in epithelial and PBMC models to restrain the progression of inflammation [39]. While Sokol et al. reported that F. prausnitzii led to significantly lower IL-12 and IFN- $\gamma$ production levels 


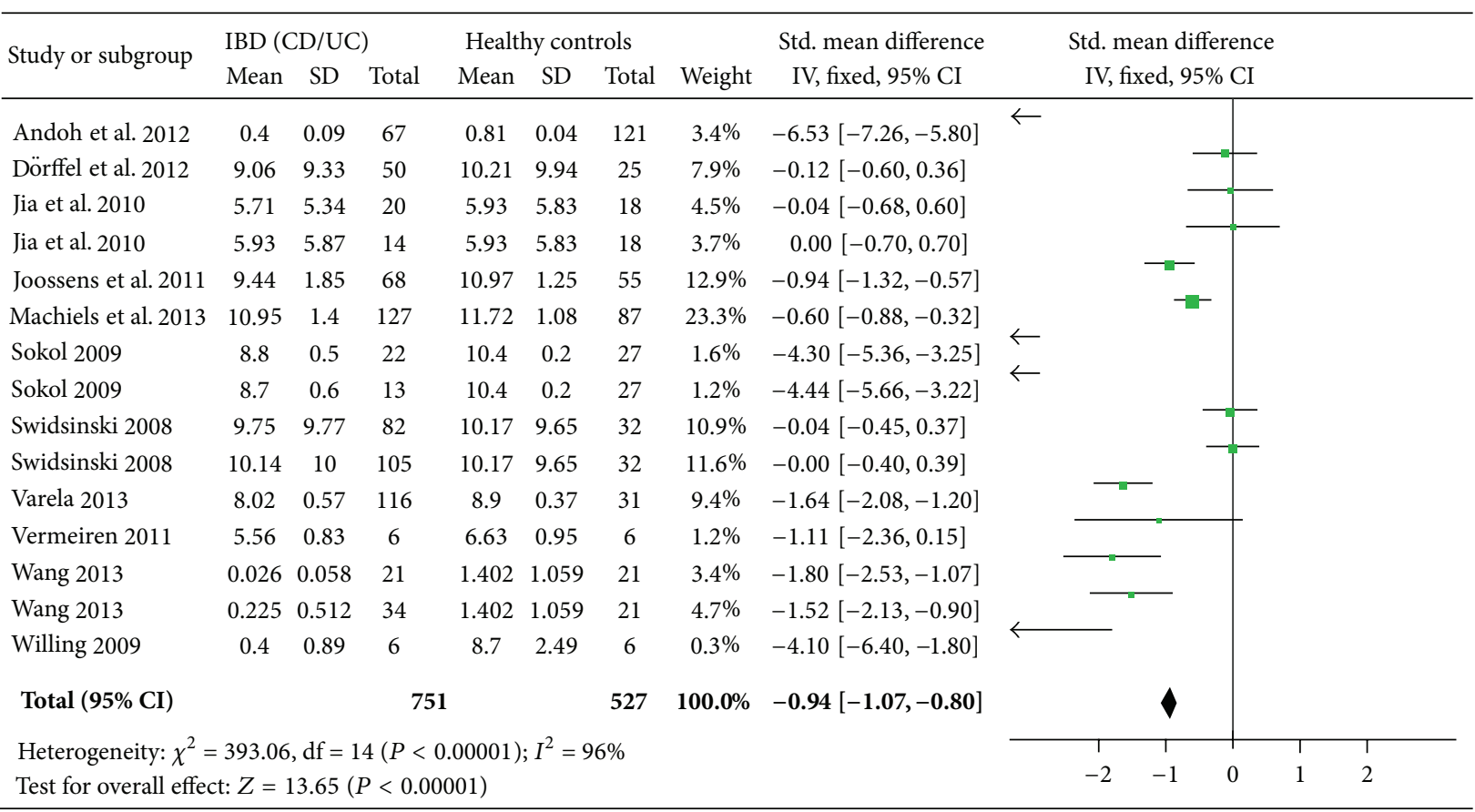

FIGURE 2: Forest plot of rate of F. prausnitzii reduction in patients with IBD versus controls.

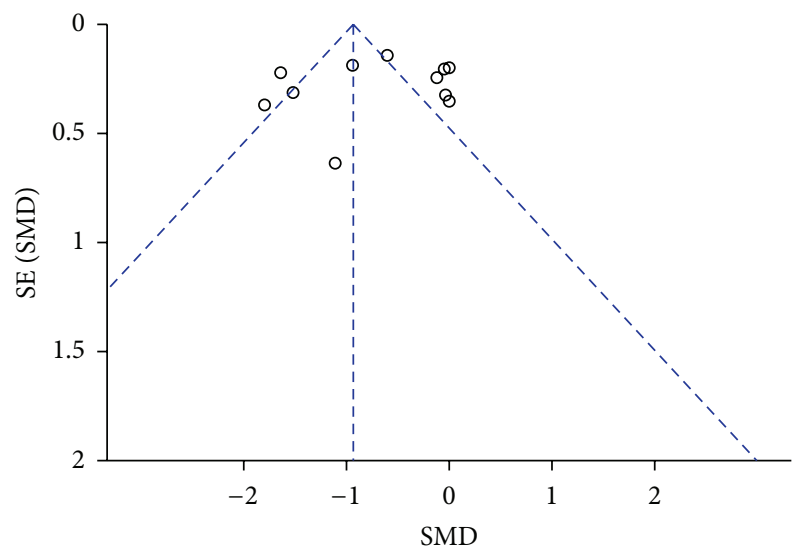

FIGURE 3: Funnel plot analysis.

and higher secretion of IL-10 in vitro peripheral blood mononuclear cells [26].

The data on the incidence of $F$. prausnitzii reduction and IBD found in the literature has several limitations. Most of the studies did not comment on the participants' previous history of treatment such as antibiotics, probiotics, or prebiotics which may influence the intestinal microbiota. It is therefore possible that study participants had been treated for dysbiosis prior to entering the study, thereby producing a false F. prausnitzii reduction. Additionally, we did not evaluate confounding factors including diet and smoking in our study, even though these factors might reduce $F$. prausnitzii levels and faecal butyrate values [40]. Furthermore, some of the included studies did not clearly identify the criteria for the IBD diagnosis. Few commented on personal review of the endoscopic findings or histology. Also we did not relate the differences in microbiota to geography and ethnicity. Lastly, most studies were performed at a single medical center.

Future studies should address these limitations. After confirming the diagnosis of IBD through the endoscopic and histological findings, PCR-DGGE, T-RFLP, or FISH for F. prausnitzii counts would be initiated. In patients found to have F. prausnitzii reduction, probiotics or prebiotics may be used to restore the "ecological balance" of intestinal microbiota. Dörffel et al. reported rifaximin was associated with an increased level of $F$. prausnitzii [9]. Other specific treatments such as infliximab and a high-dose cortisol therapy were shown to reverse the depletion of F. prausnitzii [13]. The mechanism for the inverse association between $F$. prausnitzii reduction and the initiation and perpetuation of inflammatory bowel disease has yet to be defined. Healthy controls who are age- and sex-matched to the IBD group would be selected from the same area as the IBD group and tested for $F$. prausnitzii by the same method. In both groups, a thorough history examining previous treatment such as antibiotics, probiotics, or prebiotics, steroids, 5aminosalicylates (5-ASAs), and tumor necrosis factor alpha (TNF- $\alpha$ ) antibody would be obtained. Prideaux et al. reported that regardless of ethnicity or geography, Crohn's disease resulted in reduced bacterial diversity. However, in ulcerative colitis, diversity was reduced in Chinese subjects only. It suggested that ethnicity might also play an important role in the pathogenesis of IBD [41]. 


\section{Conclusions}

In summary, our meta-analysis and systematic review suggest a possible protective benefit of $F$. prausnitzii against the development of IBD. However, significant variation among the studies and the possibility of publication bias limit the certainty of this association. Therefore, further treatment such as probiotics or prebiotics to increase the levels of $F$. prausnitzii in IBD are lead to attempts. If $F$. prausnitzii is found to indeed protect against IBD, we can approach the treatment such as supplementing the microorganisms that produce butyric acid.

\section{Conflict of Interests}

The authors declare that there is no conflict of interests regarding the publication of this paper.

\section{Authors' Contribution}

All of the authors contributed equally to the study.

\section{Acknowledgment}

The study was supported by the National Science Foundation of China (no. 81170362 and 81370508).

\section{References}

[1] M. Martinez-Medina, X. Aldeguer, F. Gonzalez-Huix, D. Acero, and L. J. Garcia-Gil, "Abnormal microbiota composition in the ileocolonic mucosa of Crohn's disease patients as revealed by polymerase chain reaction-denaturing gradient gel electrophoresis," Inflammatory Bowel Diseases, vol. 12, no. 12, pp. 1136-1145, 2006.

[2] L. Jostins, S. Ripke, R. K. Weersma et al., "Host-microbe interactions have shaped the genetic architecture of inflammatory bowel disease," Nature, vol. 491, no. 7422, pp. 119-124, 2012.

[3] B. Chassaing and A. Darfeuille-Michaud, "The commensal microbiota and enteropathogens in the pathogenesis of inflammatory bowel diseases," Gastroenterology, vol. 140, no. 6, pp. 1720-1728, 2011.

[4] A. W. Walker, J. D. Sanderson, C. Churcher et al., "Highthroughput clone library analysis of the mucosa-associated microbiota reveals dysbiosis and differences between inflamed and non-inflamed regions of the intestine in inflammatory bowel disease," BMC Microbiology, vol. 11, article 7, 2011.

[5] M. Arumugan, J. Raes, E. Pelletier et al., "Enterotypes of the human gut microbioma," Nature, vol. 473, no. 7346, pp. 174-180, 2011.

[6] P. Louis and H. J. Flint, "Diversity, metabolism and microbial ecology of butyrate-producing bacteria from the human large intestine," FEMS Microbiology Letters, vol. 294, no. 1, pp. 1-8, 2009.

[7] C. D. Packey and R. B. Sartor, "Commensal bacteria, traditional and opportunistic pathogens, dysbiosis and bacterial killing in inflammatory bowel diseases," Current Opinion in Infectious Diseases, vol. 22, no. 3, pp. 292-301, 2009.
[8] K. Machiels, M. Joossens, J. Sabino et al., "A decrease of the butyrate-producing species Roseburia hominis and Faecalibacterium prausnitzii defines dysbiosis in patients with ulcerative colitis," Gut, 2013.

[9] Y. Dörffel, A. Swidsinski, V. Loening-Baucke, B. Wiedenmann, and M. Pavel, "Common biostructure of the colonic microbiota in neuroendocrine tumors and Crohn's disease and the effect of therapy," Inflammatory Bowel Diseases, vol. 18, no. 9, pp. 16631671, 2012.

[10] K. Machiels, M. Joossens, V. de Preter et al., "Association of Faecalibacterium prausnitzii and disease activity in ulcerative colitis," Gastroenterology, vol. 140, supplement 1, article S142, no. $5,2011$.

[11] J. Luther, M. Dave, P. D. R. Higgins, and J. Y. Kao, "Association between Helicobacter pylori infection and inflammatory bowel disease: a meta-analysis and systematic review of the literature," Inflammatory Bowel Diseases, vol. 16, no. 6, pp. 1077-1084, 2010.

[12] E. Varela, C. Manichanh, M. Gallart et al., "Colonisation by Faecalibacterium prausnitzii and maintenance of clinical remission in patients with ulcerative colitis," Alimentary Pharmacology \& Therapeutics, vol. 38, no. 2, pp. 151-161, 2013.

[13] A. Swidsinski, V. Loening-Baucke, M. Vaneechoutte, and Y. Doerffel, "Active Crohn's disease and ulcerative colitis can be specifically diagnosed and monitored based on the biostructure of the fecal flora," Inflammatory Bowel Diseases, vol. 14, no. 2, pp. 147-161, 2008.

[14] J. Vermeiren, P. van den Abbeele, D. Laukens et al., "Decreased colonization of fecal Clostridium coccoides/Eubacterium rectale species from ulcerative colitis patients in an in vitro dynamic gut model with mucin environment," FEMS Microbiology Ecology, vol. 79, no. 3, pp. 685-696, 2012.

[15] M. Joossens, G. Huys, M. Cnockaert et al., "Dysbiosis of the faecal microbiota in patients with Crohn's disease and their unaffected relatives," Gut, vol. 60, no. 5, pp. 631-637, 2011.

[16] W. Wang, L. Chen, R. Zhou et al., "Increased proportion of Bifidobacterium and the Lactobacillus group and loss of butyrate-producing bacteria in inflammatory bowel disease," Journal of Clinical Microbiology, 2013.

[17] W. Jia, R. N. Whitehead, L. Griffiths et al., "Is the abundance of Faecalibacterium prausnitzii relevant to Crohn's disease?" FEMS Microbiology Letters, vol. 310, no. 2, pp. 138-144, 2010.

[18] H. Sokol, P. Seksik, J. P. Furet et al., "Low counts of Faecalibacterium prausnitzii in colitis microbiota," Inflammatory Bowel Diseases, vol. 15, no. 8, pp. 1183-1189, 2009.

[19] A. Andoh, H. Kuzuoka, T. Tsujikawa et al., "Multicenter analysis of fecal microbiota profiles in Japanese patients with Crohn's disease," Journal of Gastroenterology, vol. 47, no. 12, pp. 12981307, 2012.

[20] B. Willing, J. Halfvarson, J. Dicksved et al., "Twin studies reveal specific imbalances in the mucosa-associated microbiota of patients with ileal Crohn's disease," Inflammatory Bowel Diseases, vol. 15, no. 5, pp. 653-660, 2009.

[21] T. Fujimoto, H. Imaeda, K. Takahashi et al., "Decreased abundance of Faecalibacterium prausnitzii in the gut microbiota of Crohn's disease," Journal of Gastroenterology and Hepatology, vol. 28, no. 4, pp. 613-619, 2013.

[22] M. Galecka, P. Szachta, A. Bartnicka, L. Łykowska-Szuber, P. Eder, and A. Schwiertz, "Faecalibacterium prausnitzii and Crohn's disease-is there any connection?" Polish Journal of Microbiology, vol. 62, no. 1, pp. 91-95, 2013. 
[23] S. Miquel, R. Marín, O. Rossi et al., "Faecalibacterium prausnitzii and human intestinal health," Current Opinion in Microbiology, vol. 16, no. 3, pp. 255-261, 2013.

[24] R. Hansen, R. K. Russell, C. Reiff et al., "Microbiota of Pediatric IBD: increased Faecalibacterium prausnitzii and reduced bacterial diversity in Crohn's but not in ulcerative colitis," The American Journal of Gastroenterology, vol. 107, no. 12, pp. 19131922, 2012.

[25] C. L. O'Brien, G. E. Allison, and P. Pavli, “The more the merrier: Faecalibacterium prausnitzii in Crohn's disease," Journal of Gastroenterology and Hepatology, vol. 28, no. 5, pp. 757-759, 2013.

[26] H. Sokol, B. Pigneur, L. Watterlot et al., "Faecalibacterium prausnitzii is an anti-inflammatory commensal bacterium identified by gut microbiota analysis of Crohn disease patients," Proceedings of the National Academy of Sciences of the United States of America, vol. 105, no. 43, pp. 16731-16736, 2008.

[27] X. Qiu, M. Zhang, X. Yang, N. Hong, and C. Yu, "Faecalibacterium prausnitzii upregulates regulatory $\mathrm{T}$ cells and anti-inflammatory cytokines in treating TNBS-induced colitis," Journal of Crohn's and Colitis, vol. 7, no. 11, pp. e558-e568, 2013.

[28] M. Wang, G. Molin, S. Ahrné, D. Adawi, and B. Jeppsson, "High proportions of proinflammatory bacteria on the colonic mucosa in a young patient with ulcerative colitis as revealed by cloning and sequencing of $16 \mathrm{~S}$ rRNA genes," Digestive Diseases and Sciences, vol. 52, no. 3, pp. 620-627, 2007.

[29] S. Mondot, S. Kang, J. P. Furet et al., "Highlighting new phylogenetic specificities of Crohn's disease microbiota," Inflammatory Bowel Diseases, vol. 17, no. 1, pp. 185-192, 2011.

[30] Y. H. Siaw and A. Hart, "Commentary: is Faecalibacterium prausnitzii a potential treatment for maintaining remission in ulcerative colitis?" Alimentary Pharmacology \& Therapeutics, vol. 38, no. 5, p. 551, 2013.

[31] S. Maccaferri, B. Vitali, A. Klinder et al., "Rifaximin modulates the colonic microbiota of patients with Crohn's disease: an in vitro approach using a continuous culture colonic model system," Journal of Antimicrobial Chemotherapy, vol. 65, no. 12, pp. 2556-2565, 2010.

[32] J. L. Benjamin, C. R. Hedin, A. Koutsoumpas et al., "Smokers with active Crohn's disease have a clinically relevant dysbiosis of the gastrointestinal microbiota," Inflammatory Bowel Diseases, vol. 18, no. 6, pp. 1092-1100, 2012.

[33] S. Angelberger, W. Reinisch, A. Makristathis et al., "Temporal bacterial community dynamics vary among ulcerative colitis patients after fecal microbiota transplantation," The American Journal of Gastroenterology, vol. 108, no. 10, pp. 1620-1630, 2013.

[34] S. D. McLaughlin, A. W. Walker, C. Churcher et al., "The bacteriology of pouchitis: a molecular phylogenetic analysis using 16s rRNA gene cloning and sequencing," Annals of Surgery, vol. 252, no. 1, pp. 90-98, 2010.

[35] S. Cucchiara, V. Iebba, M. P. Conte, and S. Schippa, "The microbiota in inflammatory bowel disease in different age groups," Digestive Diseases, vol. 27, no. 3, pp. 252-258, 2009.

[36] K. E. Ashelford, N. A. Chuzhanova, J. C. Fry, A. J. Jones, and A. J. Weightman, "At least 1 in 20 16S rRNA sequence records currently held in public repositories is estimated to contain substantial anomalies," Applied and Environmental Microbiology, vol. 71, no. 12, pp. 7724-7736, 2005.

[37] S. H. Duncan, P. Louis, and H. J. Flint, "Lactate-utilizing bacteria, isolated from human feces, that produce butyrate as a major fermentation product," Applied and Environmental Microbiology, vol. 70, no. 10, pp. 5810-5817, 2004.
[38] G. T. Macfarlane and S. Macfarlane, "Fermentation in the human large intestine: its physiologic consequences and the potential contribution of prebiotics," Journal of Clinical Gastroenterology, vol. 45, supplemment, pp. S120-S127, 2011.

[39] M. E. Himmel, Y. Yao, P. C. Orban, T. S. Steiner, and M. K. Levings, "Regulatory T-cell therapy for inflammatory bowel disease: more questions than answers," Immunology, vol. 136, no. 2, pp. 115-122, 2012.

[40] R. F. J. Benus, T. S. van der Werf, G. W. Welling et al., "Association between Faecalibacterium prausnitzii and dietary fibre in colonic fermentation in healthy human subjects," British Journal of Nutrition, vol. 104, no. 5, pp. 693-700, 2010.

[41] L. Prideaux, S. Kang, J. Wagner et al., "Impact of ethnicity, geography, and disease on the microbiota in health and inflammatory bowel disease," Inflammatory Bowel Diseases, vol. 19, no. 13, pp. 2906-2918, 2013. 


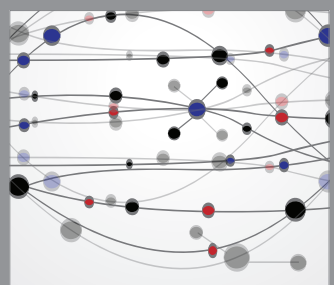

The Scientific World Journal
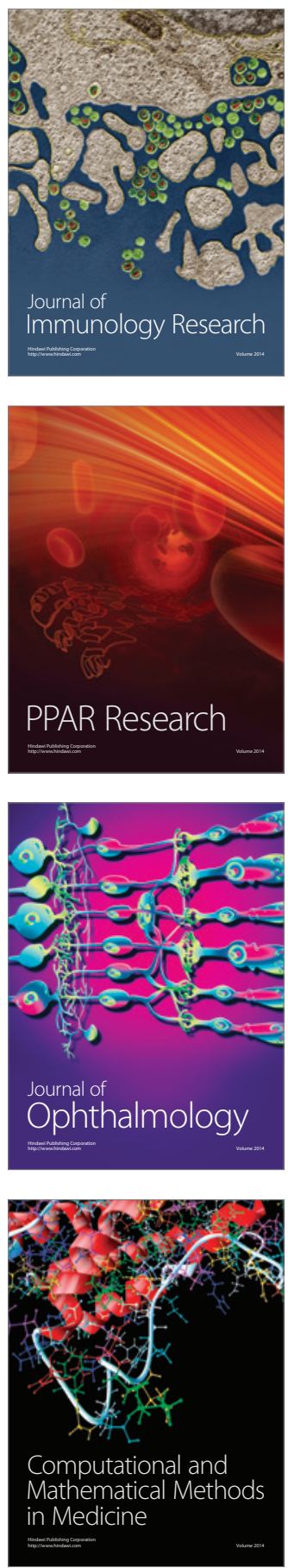

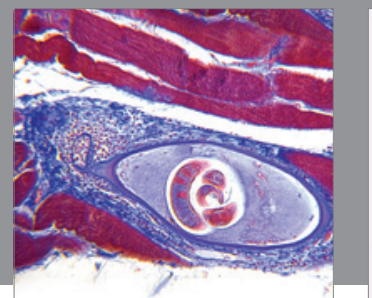

Gastroenterology

Research and Practice
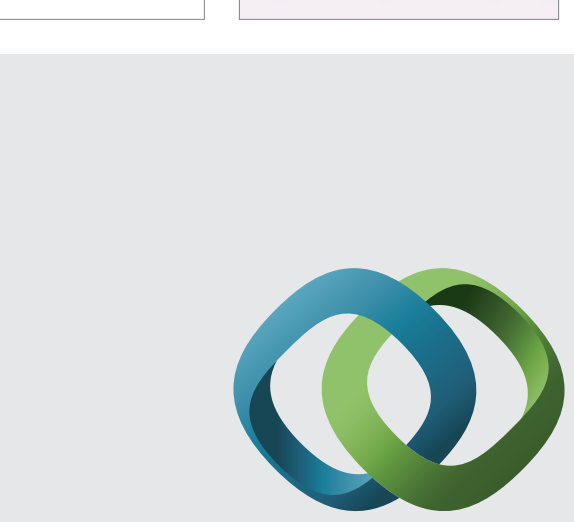

\section{Hindawi}

Submit your manuscripts at

http://www.hindawi.com
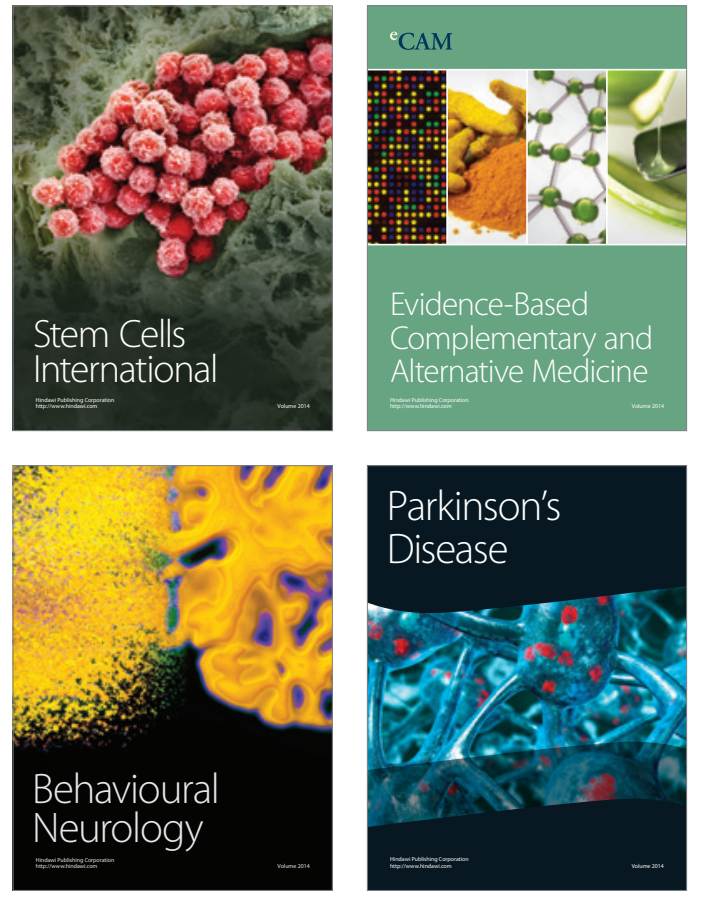
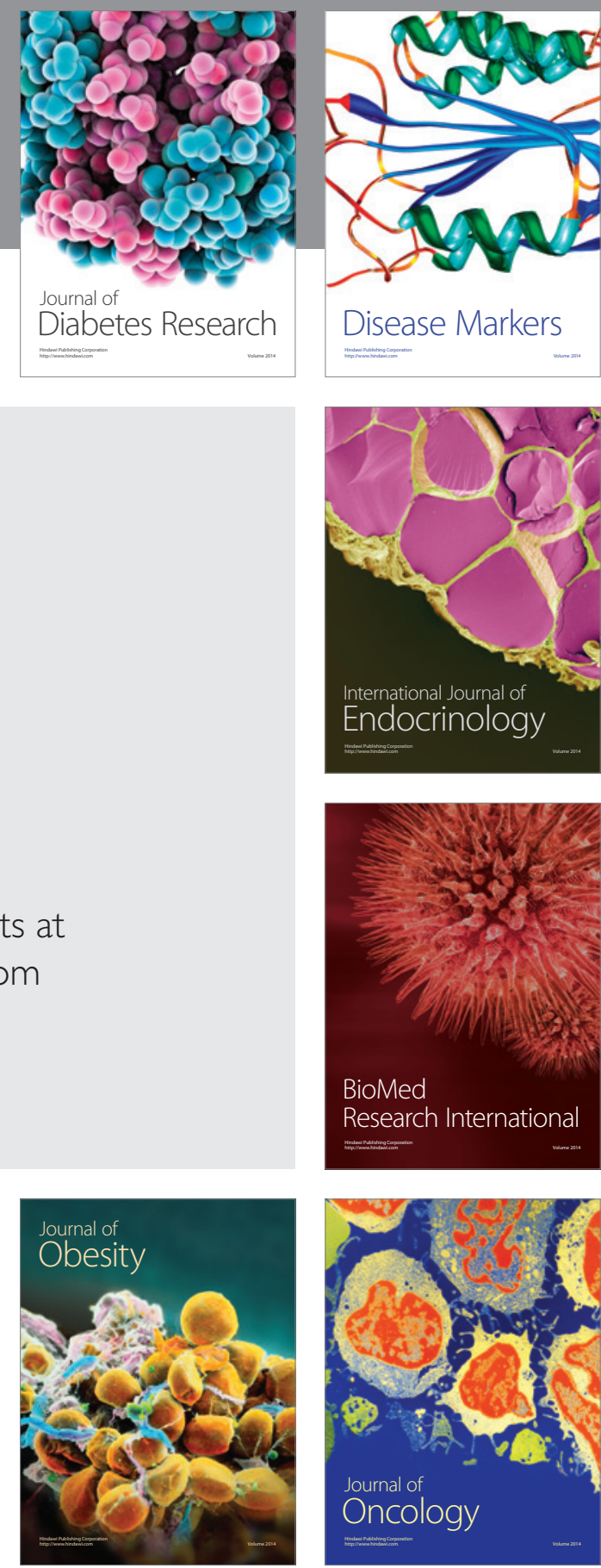

Disease Markers
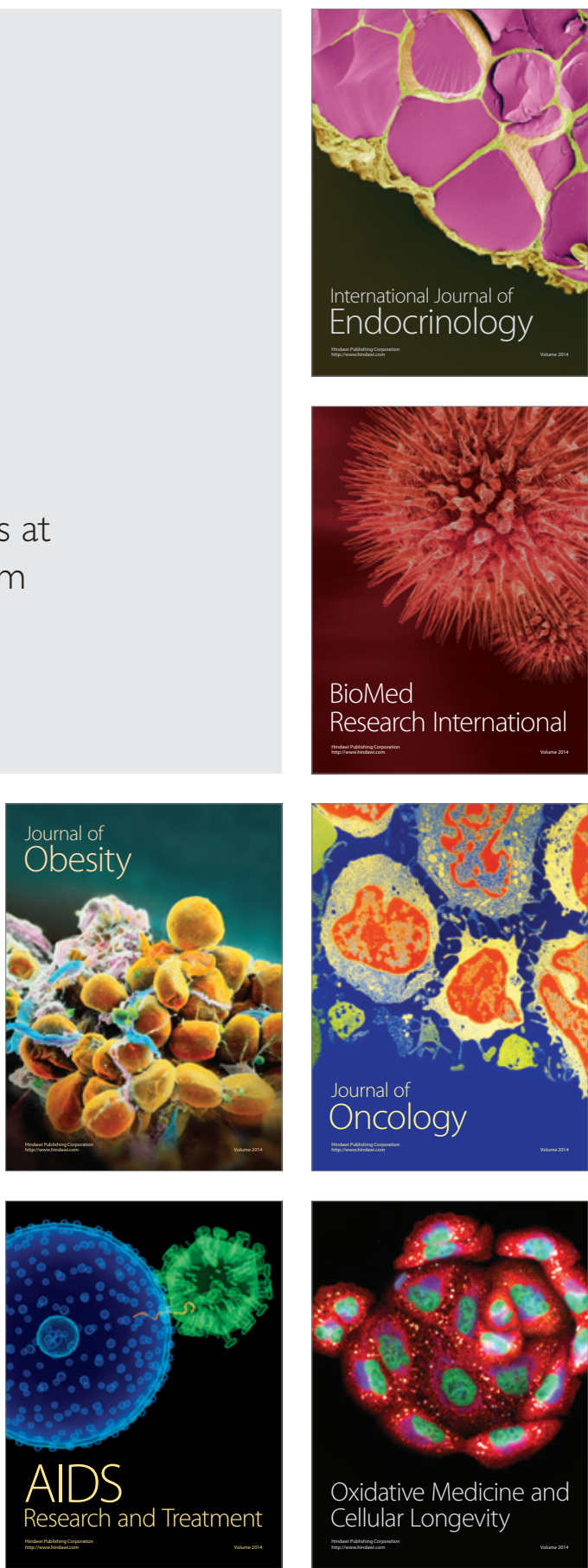